\title{
Treatment for benign thyroid nodules with a combination of natural extracts
}

\author{
FELICIAN STANCIOIU $^{1}$, DANIEL MIHAI ${ }^{2}$, GEORGIOS Z. PAPADAKIS ${ }^{3,4}$, \\ ARISTIDIS TSATSAKIS ${ }^{5}$, DEMETRIOS A. SPANDIDOS ${ }^{6}$ and CORIN BADIU ${ }^{2}$ \\ ${ }^{1}$ Department of Clinical Research, Bio-Forum Foundation, 040245 Bucharest; \\ ${ }^{2}$ Department of Thyroid Pathology, National Institute of Endocrinology CI Parhon, 011863 Bucharest, Romania; \\ ${ }^{3}$ Department of Medical Imaging, Heraklion University Hospital, Medical School, University of Crete; \\ ${ }^{4}$ Computational Biomedicine Laboratory (CBML), Institute of Computer Science (ICS), Foundation for Research and \\ Technology Hellas (FORTH); ${ }^{5}$ Centre of Toxicology Science and Research, Faculty of Medicine, \\ University of Crete; ${ }^{6}$ Laboratory of Clinical Virology, Medical School, University of Crete, 70013 Heraklion, Greece
}

Received May 13, 2019; Accepted June 19, 2019

DOI: $10.3892 / \mathrm{mmr} .2019 .10453$

\begin{abstract}
Benign thyroid nodules are among the most common endocrine disorders. Recent advances in diagnostic imaging and pathology have significantly contributed to better risk stratification of thyroid nodules. However, current treatment options, beyond surgical approaches are limited. The following placebo-controlled study presents, to the best of our knowledge, the first results of a non-invasive therapy for benign thyroid nodules. The efficacy and safety of a supplement containing spirulina, curcumin and Boswellia in euthyroid patients with benign thyroid nodules, was assessed by a 3 month, double-blind, placebo-controlled study which was completed by 34 patients. Patients with benign (FNAB documented) single thyroid nodules between 2 and $5 \mathrm{~cm}$ were evaluated in a prospective placebo-controlled cross-over trial, across 12 weeks (3 visits with six-week intervals). At each visit, the target thyroid nodule was recorded in two dimensions. In addition, plasma levels of thyroid stimulating hormone, free thyroxine and copper were assessed. The mean initial nodule area at $\mathrm{V} 1$ was $4.38 \pm 3.14 \mathrm{~cm}^{2}$, at $\mathrm{V} 23.87 \pm 2.79 \mathrm{~cm}^{2}$, and at V3 $3.53 \pm 2.84 \mathrm{~cm}^{2} ; \mathrm{P}<0.04$. Administration of the active substances $(n=34)$ was followed by a mean area decrease of $0.611 \mathrm{~cm}^{2} \pm 0.933$ (SD), while placebo administration $(\mathrm{n}=29)$ was followed by a mean decrease of $0.178 \mathrm{~cm}^{2} \pm 0.515$ (SD), $(\mathrm{P}=0.027)$. The presented findings suggest that the combination of spirulina-curcumin-Boswellia is effective in reducing the size of benign thyroid nodules. However, additional studies
\end{abstract}

Correspondence to: Dr Corin Badiu, Department of Thyroid Pathology, National Institute of Endocrinology CI Parhon, B-dul Aviatorilor 34-38, Sector 1, 011863 Bucharest, Romania E-mail: badicrin@yahoo.co.uk

Key words: spirulina, benign thyroid nodules, medical treatment are needed in order to elucidate the exact mechanisms through which the suggested supplement facilitates a decrease in the size of benign thyroid nodules.

\section{Introduction}

Thyroid nodules are a frequent pathology, with a lifetime occurrence risk in the general population estimated at 5-10\% and a gender predilection since their prevalence is higher in women (1). Furthermore, due to technological advancement and increased availability of high resolution ultrasound and other imaging modalities (e.g. incidental findings on carotid or heart ultrasound, or routine scans), in combination with the increased focus on preventive medicine and improved access to healthcare resources, their prevalence seems to be higher than estimated, with a recent study revealing thyroid nodules in about $19-68 \%$ of randomly selected individuals (1).

In regards to treatment strategies, beyond surgery, several non-invasive procedures have been developed including, radiofrequency ablation, alcohol injection, high intensityfocused ultrasound, or laser ablation. A minimally invasive procedure was recently shown to be a safe and effective treatment for benign thyroid nodules: ablation with monopolar radiofrequency (2). The last treatment approach is performed with a 16 or 18 Gauge internally cooled radiofrequency electrode after local anesthesia, which is able to treat autonomously functional nodules (3). This leads to better results for smaller and cystic nodules; post-procedural complications are present only in $17 \%$ of patients (2). Other studies have shown various results with mean decreases of $40-75 \%$ at 6 months $(4,5)$ and minimal complications in treating benign thyroid nodules with radiofrequency ablation $(5,6)$. Of note key success factors are technical skill and patient selection based on clinical evaluation (7).

Even though the vast majority (around 95\%) of thyroid nodules are benign $(1,8)$, and $5 \%$ remnant thyroid malignancies are among the most successfully treated endocrine cancers $(2,9)$, a novel, medical non-invasive treatment, which 
results in a decrease in the size of thyroid nodules would be beneficial.

\section{Materials and methods}

Patients. Participants in this clinical study were consecutive patients who presented for initial evaluation or follow-up of thyroid nodules in the outpatient department of the National Institute of Endocrinology (Bucharest, Romania) during May-September 2018 and fulfilled the inclusion and exclusion criteria.

Inclusion criteria were: Adult patients $>18$ years old; agreement to participate in the study by signing the informed consent form; euthyroid status [normal thyroid stimulating hormone (TSH) and free thyroxine (fT4) values], no active dysfunctional thyroid disease; benign thyroid nodule confirmed by FNAB (Bethesda 2); no previous thyroid surgery, thyroid ablation treatment, substitution treatment with thyroid hormones; no steroid or beta-blocker treatment; presence of one or more thyroid nodules documented by ultrasound.

Exclusion criteria were: pediatric patients ( $<18$ years old); diagnosis or suspicion of malignancy, including thyroid; autoimmune disease, including Hashimoto; Wilson's disease; hypo- or hyper-thyroidism; contraception with intrauterine device; acute infection; known allergy to spirulina, curcumin or Boswellia.

Thirty eight patients were enrolled and a total of 34 patients, 29 women and 5 men, completed the study. After being informed about the objective and requirements of the study (blood samples, ultrasound evaluations, supplement administration) with possible risks and benefits, the participants signed the informed consent form and were enrolled in the clinical study. Ethical rules established by the National Institute of Endocrinology CI Parhon according to the World Health Organisation guidelines were observed.

Study design. A double-blind, placebo-controlled, cross-over clinical trial was performed to assess the efficacy of the spirulina-based supplement in decreasing the size of thyroid nodules. The study population consisted of 34 consecutive cases randomized initially to placebo $(\mathrm{P})$ or active ingredients (AI).

There were three visits for each patient, noted as V1 - initial visit at enrollment; V2 - intermediary visit, 6 weeks post-administration of placebo $(\mathrm{P})$ or active ingredients $(\mathrm{AI})$; and V3 - final visit, after another 6 weeks of administration of $\mathrm{P}$ or AI. At each visit, each patient was tested for plasma TSH, fT4 and copper $(\mathrm{Cu})$ levels, by an independent laboratory and a thyroid ultrasound was performed by an endocrinologist (DM) using the same ultrasound machine. The nodule images and dimensions were printed for comparison.

At V1, patients were randomly selected to receive either AI or $\mathrm{P}$, so that half of them $(\mathrm{n}=17)$ received $\mathrm{P}$ and the other half $(n=17)$ received AI. Subsequently at V2, the 17 patients previously on $\mathrm{P}$ received AI, and 12 of the 17 patients previously on AI received $\mathrm{P}$, while the remaining 5 randomly chosen patients continued to receive AI. Consequently at V3, 17 patients received the sequence $\mathrm{P}-\mathrm{AI}, 12$ patients received AI-P, and 5 patients had AI-AI. The study was registered on clinicaltrials.gov site with protocol ID 'TiroNod' and ID number
NCT03535974, and titled: 'Efficacy of a spirulina supplement for Amelioration of benign thYroid nodules (ESSAY)'.

Outcomes. The primary outcome of the study was a change in size of the thyroid nodules and this is usually evaluated through a comparison of the largest diameter of the nodules between measurements via ultrasound. However, we have observed that there are patients in which the largest diameter decreases while the smallest diameter increases, and this variability may lead to significant bias in comparisons. Therefore, for more consistent results and comparisons we calculated the product of the largest (D) and smallest (d) diameters of the nodules in the same incident angle ( $\mathrm{S}=\mathrm{D} \times \mathrm{d}$ ) and compared the resulting area of the nodules measured at visits $\mathrm{V} 1,2,3$.

As secondary outcomes the serum levels of TSH, fT4 and $\mathrm{Cu}$ were assessed. The first two are considered standard evaluations of the thyroid function, while the latter was chosen for multiple reasons: i) spirulina is a significant source of $\mathrm{Cu}$ along with other minerals and vitamins; ii) most serum $\mathrm{Cu}$ is bound and transported by ceruloplasmin (10), and iii) while there is an excellent correlation between serum $\mathrm{Cu}$ and ceruloplasmin, changes of $\mathrm{Cu}$ plasma levels are faster than plasma ceruloplasmin levels, thus serum $\mathrm{Cu}$ would provide more accurate information.

Statistical analysis. NCSS 16 software was used for data analysis. Quantitative data were expressed as mean \pm standard deviation (SD). Paired sample t-test was used for comparisons of two groups (AI and P). Pearson's correlation was used to assess correlations between specific variables. Wald $\mathrm{Z}$ test for proportions was used for comparing differences in outcomes between two groups. One-way ANOVA test with the Tukey and Bonferroni post-hoc was used to compare values of TSH, fT4 and $\mathrm{Cu} . \alpha=0.05$, and $\mathrm{P}<0.05$ indicated statistical significance.

Treatment. The active treatment administered was a combination of spirulina, curcumin and Boswellia extracts, 400-50-50 mg per capsule, which were prepared alongside matching placebo capsules by DVR Pharm and BioNovative SRL. Patients were instructed to take two capsules twice daily, about $30 \mathrm{~min}$ before breakfast and lunch. Any adverse reactions were to be reported to the investigator.

Spirulina is the common generic name of about 15 species from two types of monocellular microalgae (Spirulina and Arthrospira), the most common being Arthrospira platensis (11), which grows naturally in freshwater and saltwater on all continents and in most climates, optimally in an alkaline milieu ( $\mathrm{pH}$ 9.5-12.0). It was used as the only source of nutrition in some areas in Africa for more than 1 month at a time and its first documented use was in China around 2,000 years ago even during periods of famine (12). It has a very high protein content: $40-65 \%$ of dry mass depending on species and conditions of growth/cultivation (13), and contains all essential amino acids, minerals (Fe, K, Na, Ca, P, I, Mg, Zn, $\mathrm{Cu}, \mathrm{Se}, \mathrm{Mn}, \mathrm{Cr}$ ) and vitamins (carotenoids $5 \mathrm{mg} / \mathrm{g}$ vitamins B1, B2, B3, B6, B12, folate, biotin, pantothenic acid, vit K1, $\mathrm{K} 2$ ), gamma linolenic acid, chlorophyll and phycocyanin, making it a complete food source. Alongside this remarkable composition it has important quantities of antioxidants, such 
Table I. Percentage change from initial vs. final area of thyroid nodules.

\begin{tabular}{lcccccc}
\hline $\begin{array}{l}\text { Initial vs. final area } \\
\text { decrease } \%\end{array}$ & $\begin{array}{c}<0 \\
\text { (increase) }\end{array}$ & $\begin{array}{c}\sim 0 \\
\text { (unchanged) }\end{array}$ & $\begin{array}{c}5-9.9 \% \\
\text { (decrease) }\end{array}$ & $\begin{array}{c}10-19.9 \% \\
\text { (decrease) }\end{array}$ & $\begin{array}{c}20-49.9 \% \\
\text { (decrease) }\end{array}$ & $\begin{array}{c}50-99 \% \\
\text { (decrease) }\end{array}$ \\
\hline No. of patients & 4 & 1 & 7 & 7 & 12 & 3 \\
\hline
\end{tabular}

Table II. Number of patients at V1, V2, V3, grouped by nodule area.

Initial nodule surface /

\begin{tabular}{lccccc} 
no. of patients & $0.1-0.99 \mathrm{~cm}^{2}$ & $1-2.99 \mathrm{~cm}^{2}$ & $3-5.99 \mathrm{~cm}^{2}$ & $6-8.99 \mathrm{~cm}^{2}$ & $>9 \mathrm{~cm}^{2}$ \\
\hline Visit 1 & 4 & 10 & 9 & 8 & 3 \\
Visit 2 & 5 & 10 & 12 & 5 & 2 \\
Visit 3 & 6 & 9 & 14 & 3 & 2 \\
\hline
\end{tabular}

as superoxide dysmutase $(360 \mathrm{UI} / \mathrm{g})$, zeaxanthin $(3 \mathrm{mg} / \mathrm{g})$, and vitamin E (14).

Curcumin, a natural extract from the roots of Curcuma longa, has also been in use for centuries in Asia for various ailments. It is a potent anti-inflammatory substance with pleiotropic actions, including antioxidant and metabolic modulation. Its effects are well studied, with multiple clinical trials showing benefits in arthritis, inflammatory bowel disease, prediabetes, and even in early stages of neoplastic disease (15). A recent meta-analysis on 15 randomized clinical trials shows that curcumin downregulates inflammation and oxidation products by reducing levels of IL-6, hs-CRP, and MDA (16).

Boswellia is also a potent anti-inflammatory molecule, which blocks the NFkB pathway and modulates the activity of regulatory and effector T cells (17). Together with curcumin it was shown to inhibit production of inflammatory cytokines Il-6, IL-8, TNF- $\alpha$ and reactive oxygen species $(18,19)$.

\section{Results}

Patients. A total of 38 patients were enrolled of whom 34 patients, 29 women and 5 men, aged $53.44 \pm 12.63$ years, completed the study and were evaluated for the primary outcome - the size of the thyroid nodules evaluated by ultrasound at 1st (initial), 2nd (intermediate) and 3rd (final) patient visits. These 34 patients were also tested for serum TSH, fT4 and $\mathrm{Cu}$.

The overall reduction in the measured surface of the thyroid nodules for all the 34 study participants, calculated as the mean percentage decrease in the surface of nodules, was $20.3 \% \pm 19.18 \%$ (SD). For all 34 patients, mean initial nodule area at initial visit (V1 S) was $4.38 \pm 3.14 \mathrm{~cm}^{2}$; at 2nd visit (V2 S) mean nodule area and SD was: $3.87 \pm 2.79 \mathrm{~cm}^{2}$; and at 3rd visit (V3 S) mean nodule area and SD was $3.53 \pm 2.84 \mathrm{~cm}^{2}$.

Thyroid nodule size and modifications. From the total of 34 patients who completed the 3 ultrasound evaluations, 29 patients $(85.29 \%)$ had decreases in the areas of thyroid nodules of $5 \%$ or more at the end of the study, and 22 patients $(64.7 \%)$ had decreases of $10 \%$ or more of the thyroid nodule area after 3 months.

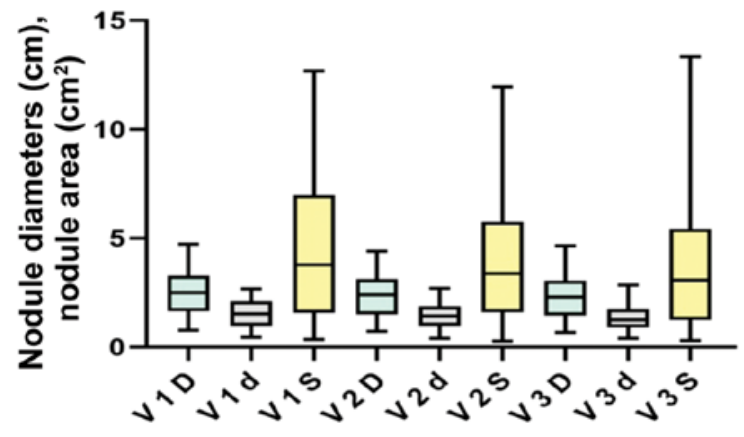

Figure 1. Nodule size at V1, V2, V3 in all patients. In all 34 patients, the mean initial nodule area at initial visit (V1 S) was $4.38 \pm 3.14 \mathrm{~cm}^{2}$, at 2 nd visit (V2 S) mean nodule area and SD was: $3.87 \pm 2.79 \mathrm{~cm}^{2}$, and at $3 \mathrm{nd}$ visit (V3 S) mean nodule area and SD was $3.53 \pm 2.84 \mathrm{~cm}^{2}$. D, large diameter; $\mathrm{d}$, small diameter; $\mathrm{S}$, area; SD, standard deviation.

One patient had insignificant decrease in nodule area $(0.21 \%)$, and 4 patients had increases in the size of the nodules of $1.66,2.36,5.13$ and $23.81 \%$, respectively, at the final ultrasound assessment (Table I).

Patients were also grouped according to the initial area of the nodules and their modifications at V2 and V3.

Table II summarizes the modifications in nodule areas and shows an increase in the number of patients with nodule areas of $<6 \mathrm{~cm}^{2}$ at study visits 2 and 3 compared to V1 due to a gradual decrease in nodule areas. Patients with larger thyroid nodules $\left(>6 \mathrm{~cm}^{2}\right)$ were the most dynamic group, their number decreasing from V1-V2-V3 to 11-7-5, respectively.

Nodule size and visits. A graphic comparison of the effects of all patients at the three study visits is given in Fig. 1, which shows that the decrease of the mean areas of the nodules between the three visits were statistically significant: for V1 S vs. V2 S the paired sample t-test has $\mathrm{P}=0.00006$; for V2 S vs. V3 S the paired sample t-test has $\mathrm{P}=0.04182$; and for V1 S vs. V3 S paired sample t-test has $\mathrm{P}=0.00008$.

A comparison of the effects of the active substance vs. placebo on decreasing the surface of thyroid nodules showed that after the initial administration of the placebo and active 
Table III. Decrease in thyroid nodules area after active substances and placebo.

\begin{tabular}{lcccc}
\hline Mean \pm SD decrease & Active substance & Placebo & Statistical significance & Total active + placebo \\
\hline $\mathrm{V} 2-\mathrm{V} 1, \mathrm{~cm}^{2}$ & $0.784 \pm 0.708$ & $0.238 \pm 0.444$ & $\mathrm{P}=0.017^{\mathrm{a}}$ & $0.511 \pm 0.645$ \\
$\mathrm{~V} 3-\mathrm{V} 2, \mathrm{~cm}^{2}$ & $0.499 \pm 1.069$ & $0.094 \pm 0.612$ & $\mathrm{P}=0.16^{\mathrm{a}}$ & $0.343 \pm 0.944$ \\
$\mathrm{~V} 3-\mathrm{V} 1, \mathrm{~cm}^{2}$ & $0.611 \pm 0.933$ & $0.178 \pm 0.515$ & $\mathrm{P}=0.027^{\mathrm{a}}$ & $0.854 \pm 1.108$ \\
Patients nb V2 vs. V1 & $16 / 17$ & $13 / 17$ & $\mathrm{P}=0.14^{\mathrm{b}}$ & $29 / 34$ \\
Patients nb V3 vs. V2 & $18 / 22$ & $6 / 12$ & $\mathrm{P}=0.051^{\mathrm{b}}$ & $23 / 34$ \\
Patients nb V3 vs. V1 & $34 / 39$ & $19 / 29$ & $\mathrm{P}=0.033^{\mathrm{b}}$ & $30 / 34$ \\
\hline
\end{tabular}

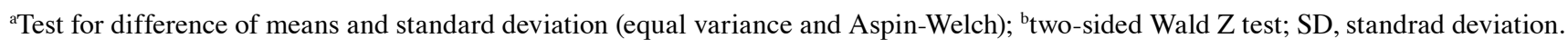

substances, the mean decrease with AI was $19.88 \pm 15.55 \%$, while with the placebo the decrease was less, $9.31 \% \pm 14.29 \%$. The two-sided t-test for the two groups had a t value of 2.03 , and the difference between placebo and active substances was marginally statistically significant $(\mathrm{P}=0.047)$.

Consequently, we used two additional statistical tests: the Wald $\mathrm{Z}$ test to compare the proportion of patients with modifications in nodule areas, grouped in AI vs. P and also tested both the equal variance and Aspin-Welch variants for the difference of means \pm SD between the AI and P groups.

Decrease in thyroid nodules area after active substances and placebo. The difference in the proportion of patients with decreased thyroid nodule areas on active substances vs. placebo groups was directly increased with each visit and the number of patients on AI vs. P; it achieved statistical significance at V3 vs. V1 $(\mathrm{P}=0.033)$; statistically significant results were obtained by comparing the mean $\pm \mathrm{SD}$ of nodule areas in $\mathrm{AI}$ and $\mathrm{P}$ groups at $\mathrm{V} 3-\mathrm{V} 1(\mathrm{P}=0.027)$ and $\mathrm{V} 2-\mathrm{V} 1$ $(\mathrm{P}=0.017)$, but not V3-V2 $(\mathrm{P}=0.16)$, probably because the AI effect was still present in patients taking $\mathrm{P}$ in the sequence AI-P (Table III).

Comparing the overall effects of the placebo and the active substances on all administrations (39 active substances, 29 placebo, total 68 sequences in 34 patients, Fig. 2), we have observed that the administration of the active substances $(n=39)$ was followed by a mean decrease in nodule areas of $0.611 \mathrm{~cm}^{2} \pm 0.933$ (SD), while placebo administration $(\mathrm{n}=29)$ was followed by a mean decrease of $0.178 \mathrm{~cm}^{2} \pm 0.515$ (SD). Testing for statistical significance between the two groups (active and placebo) at V3 versus V1 with t-test for means and standard deviation, there was a mean difference of $0.433 \mathrm{~cm}^{2}$, which is statistically significant both when calculated with the equal variance t-test (standard error of the difference of 0.192 , $\mathrm{P}=0.027$ ) and the unequal variance Aspin-Welch test (standard error of the difference of $0.177, \mathrm{P}=0.017$ ) (Table III).

Based on these data it is evident that the active combination is effective in reducing the size of the thyroid nodules, even though it was administered for only 6 weeks out of the total of 3 months.

Values of TSH, fT4 and Cu at the three visits. Beyond efficacy, we have also monitored the influence of treatment on thyroid function and the correlation with $\mathrm{Cu}$ levels. To determine whether the active substances or the placebo had a significant

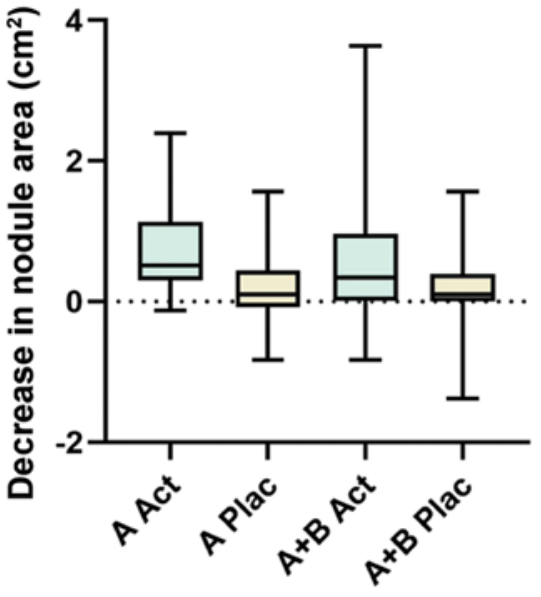

Figure 2. The figure graphically represents the significant data from Table III, comparing the means, min and max values nodule areas in the AI (Act) and $\mathrm{P}$ (Plac) groups after the initial 6 week administration of AI or P (V2-V1, noted 'A') and after 12 weeks of AI or P (V3-V1, noted 'A+B', A being the initial 6 weeks, B the final 6 weeks of study). As noted in Table III, in the 'B' period of the study (V3-V2) the mean nodule areas in the AI vs. P groups were not statistically significant. A Act, A Plac $(n=17), A+B$ Act $(n=39), A+B$ Plac $(n=29)$.

effect on thyroid function, we compared the respective values of TSH and fT4 for all 3 visits (visit 1 vs. 2, visit 1 vs. 3 and 2 vs. 3) with the one-way ANOVA test and the Tukey and Bonferroni post-hoc tests. There was no statistically significant difference between the respective values of TSH or fT4 in the initial, intermediary or final visits. A similar result was obtained when comparing the levels of serum $\mathrm{Cu}$ between the 3 visits; there were no significant differences between the respective values (Table IV).

To verify the interdependence of the four variables measured in this study: nodule surface, TSH, fT4 and $\mathrm{Cu}$ levels, we calculated the Pearson's correlation indices and found that the strongest correlation was between the decrease in nodules surface and TSH levels at visits 2 and 3: $\Delta \mathrm{S} 2-\mathrm{S} 3$ and $\Delta$ TSH2 - TSH3, Pearson's correlation $=0.52, \mathrm{P}=0.002$. There was also a significant inverse correlation of serum $\mathrm{Cu}$ and TSH modifications between the initial and final visits: Pearson's correlation of $\Delta \mathrm{Cu}$ V1-V3 and $\Delta \mathrm{TSH}$ V1-V3 was $\mathrm{r}=-0.3885$; with $\mathrm{P}=0.033$ (data not shown).

Adverse effects. Four patients withdrew from the study before completion. Three of them reconsidered participation 
Table IV. Values of TSH, fT4 and $\mathrm{Cu}$ at the three visits, and ANOVA test values.

\begin{tabular}{lccccccccc}
\hline Visit and test type & V1 TSH & V2 TSH & V3 TSH & V1 fT4 & V2 fT4 & V3 fT4 & V1 Cu & V2 Cu & V3Cu \\
\hline Number tested & 34 & 34 & 32 & 33 & 34 & 32 & 33 & 33 & 31 \\
Mean & 1.50 & 1.37 & 1.30 & 1.18 & 1.18 & 1.196 & 127.65 & 127.13 & 128.38 \\
SD & 1.27 & 1.11 & 0.96 & 0.17 & 0.17 & 0.16 & 21.55 & 21.34 & 16.26 \\
ANOVA V1-V2-V3 & F-ratio $=0.28472$, P=0.75 & F-ratio $=0.06792$, P=0.93 & F-ratio $=0.03162, \mathrm{P}=0.96$
\end{tabular}

TSH, thyroid stimulating hormone; fT4, free thyroxine; $\mathrm{Cu}$, copper.

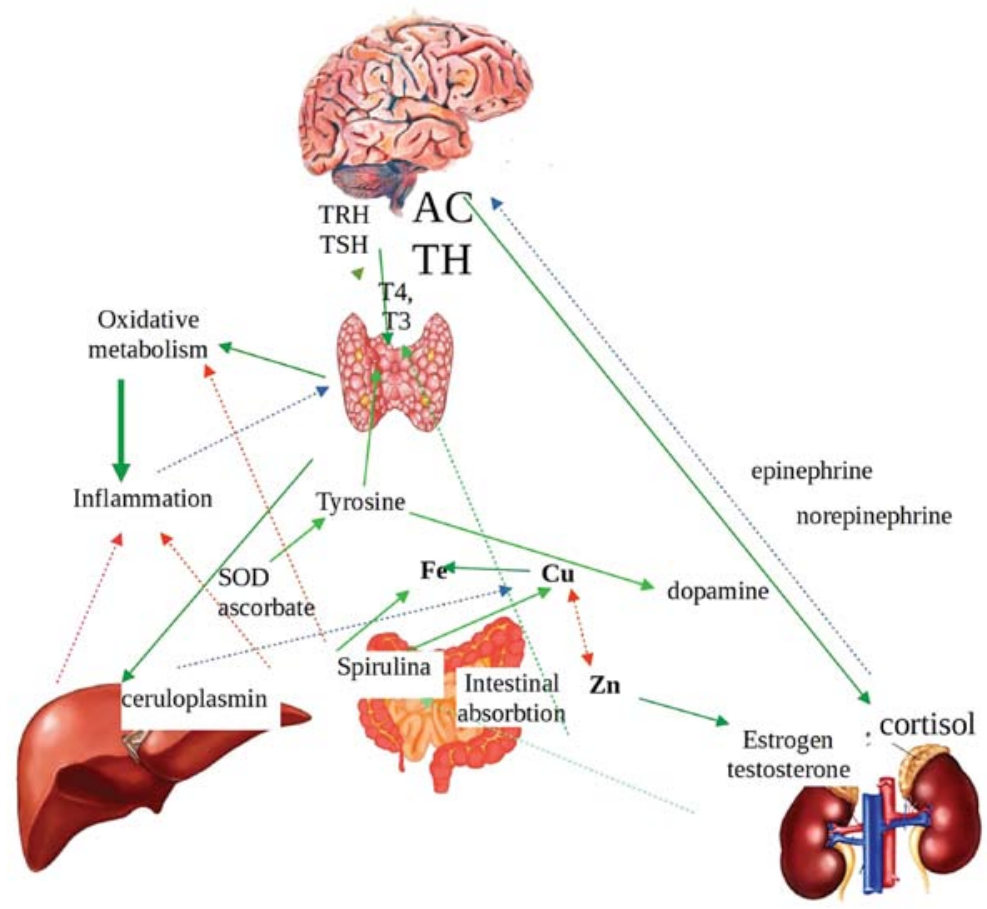

Figure 3. Interactions between thyroid hormones, ceruloplasmin, adrenal hormones, and processes of intestinal absorbtion, oxidative metabolism and inflammation. T3/T4, triiodothyronine/thyroxine; TRH/TSH, thyroid releasing/stimulating hormones; ACTH, adrenocorticotropic hormone; SOD, superoxide dysmutase.

due to personal reasons, while one patient stopped one week post-administration of the study substances, because of development of a rash on the trunk and upper portions of limbs, while on multiple medications. Following investigation, it was revealed that the type of substance administered to the patient was the placebo.

No other adverse effects were recorded on the study participants for either the active substances or the placebo.

\section{Discussion}

The link between thyroid function and ceruloplasmin, the most important $\mathrm{Cu}$-based enzyme, was observed a few decades ago, with a significant elevation of serum ceruloplasmin being observed in patients on oral contraceptives together with an increase in thyroxine binding index, while free thyroxine levels were unchanged (20). Similarly, in male rats estradiol elevated ceruloplasmin levels, while testosterone and thyroid hormones inhibited this action (21). Furthermore, it was shown that administration of L-thyroxine suppresses the stimulation of ceruloplasmin by estrogen administration (22). Opposite modifications of ceruloplasmin levels in hypo- and hyperthyroid patients, in tandem with thyroid function, as well as oxidation status (malonyldialdehyde) and uric acid levels were observed by a Romanian team (23). New direct links between ceruloplasmin, oxidative stress and inflammation were recently shown with TNF-related apoptosis-inducing ligand (TRAIL) down-regulating ceruloplasmin expression in inflammation (24), and modulation of TNF- $\alpha$-induced neutrophil apoptosis by ceruloplasmin (25). Low levels of ceruloplasmin were shown in a hypothyroid patient with Hashimoto thyroiditis (26).

Recent findings have shown that thyroid hormones increase serum $\mathrm{Cu}$ levels by stimulating the synthesis of ceruloplasmin in the liver as reflected in the mRNA levels, and this action is abolished in cases of hyperthyroidism associated with receptor resistance to thyroid hormones (27). In addition, the ratio of serum $\mathrm{Cu}$ and selenium was proposed as an indirect marker for receptor resistance to thyroid hormones. Stimulation of hepatic ceruloplasmin mRNA 
by thyroxine and dexamethasone was previously shown in rats (28). It was also found that a $\mathrm{Cu}$-deficient diet increases the levels of serum free triiodothyronine but not fT4 in rats (29); similarly a strong link between serum T3 and $\mathrm{Cu}$ was demonstrated in rat pups (30). $\mathrm{Cu}$ and $\mathrm{Zn}$ levels in serum and tissue are modulated by thyroid hormones as shown by thyroparathyroidectomy and T4 replacement in rat. Interestingly, it was seen that ceruloplasmin is present in follicular carcinoma and absent in adenomas (31-33). Further differences in the oxidative and inflammatory processes involved in benign and malignant thyroid nodules versus normal thyroid tissue (hypoxia-inducible factor-1, complement factor D, matrix metalloprotease 1, Von Willebrand Factor - were shown in different studies (34) The interrelations between thyroid hormones, ceruloplasmin, adrenal hormones and important metabolic processes are presented in Fig. 3.

Before administration of the indicated spirulinacurcumin-Boswellia combination, a thorough evaluation by an DM (with or without a thyroid ultrasound) should be performed, in order to establish euthyroid status, exclude patients with suspicion of malignancy, acute infection, autoimmune disease, Wilson's disease, and monitor possible associations with contraceptives or other simultaneous medical treatments.

In summary, the combination of spirulina-curcuminBoswellia is effective in reducing the size of benign thyroid nodules and can be safely administered in the doses used in the presented clinical study. The mechanism by which the decrease of the nodule size is facilitated, is probably linked to the anti-inflammatory effects, the improvement of the antioxidant status and the ceruloplasmin actions. However, additional studies are needed in order to elucidate these associations, which may also provide important insights into the pathogenesis of thyroid nodules.

\section{Acknowledgements}

Not applicable.

\section{Funding}

This study was financed by a grant received by The Bio-Forum Foundation from DVR Pharm SRL and BioNovativ SRL which intend to manufacture and bring to market the spirulina-curcumin-boswellia combination under the name "TiroDren".

\section{Availability of data and materials}

The data sets used and/or analyzed during the current study are available from the corresponding author on reasonable request.

\section{Authors' contributions}

FS, DM and CB participated in data collection and analysis. FS, GZP, AT and DAS participated in the design of the study. FS, DM and GZP participated in the writing of the manuscript. FS, AT and CB participated in data interpretation. All authors read and approved the final manuscript.

\section{Ethics approval and consent to participate}

All participants provided written informed consent for the whole study. The study observed the ethical rules on clinical studies established by the National Institute of Endocrinology CI Parhon (Bucharest, Romania) according to the World Health Organisation guidelines.

\section{Patient consent for publication}

Not applicable.

\section{Competing interest}

DAS is the Editor-in -Chief for the journal, but had no personal involvement in the reviewing process, or any influence in terms of adjudicating on the final decision, for this article. The other authors declare that they have no competing interests. Bio-Forum Foundation from DVR Pharm SRL and BioNovativ SRL intend to manufacture and bring to market the spirulina-curcumin-boswellia combination under the name 'TiroDren'.

\section{References}

1. Haugen BR, Alexander EK, Bible KC, Doherty GM, Mandel SJ, Nikiforov YE, Pacini F, Randolph GW, Sawka AM, Schlumberger M, et al: 2015 American thyroid association management guidelines for adult patients with thyroid nodules and differentiated thyroid cancer: The American thyroid association guidelines task force on thyroid nodules and differentiated thyroid cancer. Thyroid 26: 1-133, 2016.

2. Burman KD and Wartofsky L: CLINICAL PRACTICE. Thyroid Nodules. N Engl J Med 373: 2347-2356, 2015.

3. Bernardi S, Stacul F, Michelli A, Giudici F, Zuolo G, de Manzini N, Dobrinja C, Zanconati F and Fabris B: 12-month efficacy of a single radiofrequency ablation on autonomously functioning thyroid nodules. Endocrine 57: 402-408, 2017.

4. Cesareo R, Palermo A, Pasqualini V, Simeoni C, Casini A, Pelle G, Manfrini S, Campagna G and Cianni R: Efficacy and safety of a single radiofrequency ablation of solid benign non-functioning thyroid nodules. Arch Endocrinol Metab 61: 173-179, 2017.

5. Wu W, Gong X, Zhou Q, Chen X and Chen X: Ultrasoundguided percutaneous microwave ablation for solid benign thyroid nodules: Comparison of MWA versus control group. Int J Endocrinol 2017: 9724090, 2017.

6. Yue W, Wang S, Wang B, Xu Q, Yu S, Yonglin Z and Wang X: Ultrasound guided percutaneous microwave ablation of benign thyroid nodules: Safety and imaging follow-up in 222 patients. Eur J Radiol 82: e11-e16, 2013.

7. Dobnig $\mathrm{H}$ and Amrein K: Monopolar radiofrequency ablation of thyroid nodules: A prospective Austrian single-center study. Thyroid 28: 472-480, 2018.

8. Durante C, Costante G, Lucisano G, Bruno R, Meringolo D, Paciaroni A, Puxeddu E, Torlontano M, Tumino S, Attard M, et al: The natural history of benign thyroid nodules. JAMA 313: 926-935, 2015.

9. Hay ID, Thompson GB, Grant CS, Bergstralh EJ, Dvorak CE, Gorman CA, Maurer MS, McIver B, Mullan BP, Oberg AL, et al: Papillary thyroid carcinoma managed at the Mayo Clinic during six decades (1940-1999): Temporal trends in initial therapy and long-term outcome in 2444 consecutively treated patients. World J Surg 26: 879-885, 2002.

10. Twomey PJ, Viljoen A, House IM, Reynolds TM and Wierzbicki AS: Relationship between serum copper, ceruloplasmin, and non-ceruloplasmin-bound copper in routine clinical practice. Clin Chem 51: 1558-1559, 2005.

11. Habib, M Ahsan B, Huntington T and Hasan MA: A review on culture, production and use of Spirulina as food for humans and feeds for domestic animals and fish. In: FAO Fisheries and Aquaculture Circular No.1034. Food and Agriculture Organization of the United Nations, Rome, Italy, 2008. 
12. Spolaore P, Joannis-Cassan C, Duran E and Isambert A Commercial applications of microalgae. J Biosci Bioeng 101: 87-96, 2006.

13. Becker EW: Micro-algae as a source of protein. Biotechnol Adv 25: 207-210, 2007.

14. Kent M, Welladsen HM, Mangott A and Li Y: Nutritional evaluation of Australian microalgae as potential human health supplements. PLoS One 10: e0118985, 2015.

15. Perrone D, Ardito F, Giannatempo G, Dioguardi M, Troiano G, Lo Russo L, DE Lillo A, Laino L and Lo Muzio L: Biological and therapeutic activities, and anticancer properties of curcumin. Exp Ther Med 10: 1615-1623, 2015.

16. Tabrizi R, Vakili S, Akbari M, Mirhosseini N, Lankarani KB, Rahimi M, Mobini M, Jafarnejad S, Vahedpoor Z and Asemi Z: The effects of curcumin-containing supplements on biomarkers of inflammation and oxidative stress: A systematic review and meta-analysis of randomized controlled trials. Phytother Res 33: 252-262, 2019.

17. Beghelli D, Isani G, Roncada P, Andreani G, Bistoni O, Bertocchi M, Lupidi G and Alunno A: Antioxidant and ex vivo immune system regulatory properties of Boswellia serrata extracts. Oxid Med Cell Longev 2017: 7468064, 2017.

18. Governa P, Marchi M, Cocetta V, De Leo B, Saunders PTK, Catanzaro D, Miraldi E, Montopoli M and Biagi M: Effects of Boswellia Serrata Roxb. and Curcuma longa L. in an in vitro intestinal inflammation model using immune cells and Caco-2. Pharmaceuticals (Basel) 11: E126, 2018.

19. Kizhakkedath R: Clinical evaluation of a formulation containing Curcuma longa and Boswellia serrata extracts in the management of knee osteoarthritis. Mol Med Rep 8: 1542-1548, 2013.

20. Schenk H, Haralambus I, Leb G, Pickel H and Goebel R: The effect of oral contraceptives on levels of thyroid hormone, blood coagulation and ceruloplasmin. MMW Munch Med Wochenschr 119: 941-944, 1977 (In German).

21. Schreiber V and Pribyl T: Effect of interaction of oestrogen, testosterone and thyroid hormones on the serum ceruloplasmin level in rats. Physiol Bohemoslov (2): 129-137, 1977.

22. Schreiber V, Pribyl T and Jahodová J: Reactions of hypothalamic ascorbic acid, serum ceruloplasmin and the adenohypophysis to oestradiol: Inhibition by L-thyroxine. Physiol Bohemoslov 29: 11-20, 1980.

23. Dumitriu L, Bartoc R, Ursu H, Purice M and Ionescu V: Significance of high levels of serum malonyl dialdehyde (MDA) and ceruloplasmin (CP) in hyper- and hypothyroidism. Endocrinologie 26: 35-38, 1988.

24. Tisato V, Gallo S, Melloni E, Celeghini C, Passaro A, Zauli G, Secchiero P, Bergamini C, Trentini A, Bonaccorsi G, et al: TRAIL and ceruloplasmin inverse correlation as a representative crosstalk between inflammation and oxidative stress. Mediators Inflamm 2018: 9629537, 2018.
25. Golenkina EA, Viryasova GM, Galkina SI, Gaponova TV, Sud'ina GF and Sokolov AV: Fine regulation of neutrophil oxidative status and apoptosis by ceruloplasmin and its derivatives. Cells 7: E8, 2018

26. Valenzise M, Porcaro F, Zirilli G, De Luca F, Cinquegrani M and Aversa T: Hypoceruloplasminemia: An unusual biochemical finding in a girl with Hashimoto's thyroiditis and severe hypothyroidism. Pediatr Med Chir 40: 2018. https://doi. org/10.4081/pmc.2018.179.

27. Mittag J, Behrends T, Nordström K, Anselmo J, Vennström B and Schomburg L: Serum copper as a novel biomarker for resistance to thyroid hormone. Biochem J 443: 103-109, 2012.

28. Fitch CA, Song Y and Levenson CW: Developmental regulation of hepatic ceruloplasmin mRNA and serum activity by exogenous thyroxine and dexamethasone. Proc Soc Exp Biol Med 221: 27-31, 1999.

29. Kralik A, Kirchgessner M and Eder K: Concentrations of thyroid hormones in serum and activity of hepatic 5 monodeiodinase in copper-deficient rats. Z Ernahrungswiss 35: 288-291, 1996.

30. Bastian TW, Lassi KC, Anderson GW and Prohaska JR: Maternal iron supplementation attenuates the impact of perinatal copper deficiency but does not eliminate hypotriiodothyroninemia nor impaired sensorimotor development. J Nutr Biochem 22: 1084-1090, 2011

31. Song B: Immunohistochemical demonstration of epidermal growth factor receptor and ceruloplasmin in thyroid diseases. Acta Pathol Jpn 41: 336-343, 1991.

32. Kondi-Pafiti A, Smyrniotis V, Frangou M, Papayanopoulou A Englezou M and Deligeorgi H: Immunohistochemical study of ceruloplasmin, lactoferrin and secretory component expression in neoplastic and non-neoplastic thyroid gland diseases. Acta Oncol 39: 753-756, 2000.

33. Vaideeswar P, Pandit AA, Khilnani PH and Powar HS Differentiation of follicular adenoma and carcinoma of thyroid by immunohistochemical demonstration of ceruloplasmin. Indian J Pathol Microbiol 37: 165-169, 1994.

34. Wang Q, Shen Y, Ye B, Hu H, Fan C, Wang T, Zheng Y, Lv J, $\mathrm{Ma} \mathrm{Y}$ and Xiang $\mathrm{M}$ : Gene expression differences between thyroid carcinoma, thyroid adenoma and normal thyroid tissue. Oncol Rep 40: 3359-3369, 2018.

This work is licensed under a Creative Commons Attribution-NonCommercial-NoDerivatives 4.0 International (CC BY-NC-ND 4.0) License. 